\title{
EFFECT OF DRIVING BREAKS AND 34-HOUR RECOVERY PERIOD ON MOTOR CARRIER CRASH ODDS
}

\author{
Kun-Feng Wu \& Paul Jovanis \\ Pennsylvania State University \\ University Park, Pennsylvania, USA \\ Email: kxw930@psu.edu
}

\begin{abstract}
Summary: This research seeks to contribute to our knowledge of the relationship between truck driver hours of service and motor carrier crash odds. Data were collected from less-than-truckload carriers in 2004-05 and 2010 including the precise hours of service for crash-involved drivers and a random sample of noncrash involved drivers. Time-dependent logistic regression models were formulated to study the probability of a crash after a certain number of hours driving, given survival until that time. In addition to driving time during a trip, the models included presence of 34 hours consecutively off-duty immediately prior to the trip of interest and the use of breaks from driving by the driver. Multi-day driving patterns, developed using cluster analysis, cover the 7 days prior to the day of interest in an attempt to capture the effect of the pattern of driving over many days. Among the findings of this research are: (1) Driving hours 6 through 11 show continuous increases in the crash risk, (2) substantial and consistent benefits for drivers who take breaks compared to drivers who drive without breaks; benefits ranged from 34 to 47 percent reduction in crash odds, depending on the number of breaks taken, (3) drivers who had 34 hours or more off-duty immediately prior to the measurement period had a nearly 43 percent increase in crash odds, and (4) additional investigation shows that drivers have the greatest difficulty immediately after returning from the extended time off; the effect then diminishes with time.
\end{abstract}

\section{INTRODUCTION}

It is generally accepted that truck driver safety is associated with the driver's work schedule including driving time, on-duty not driving time, and off-duty time. In 1938, the now-abolished Interstate Commerce Commission (ICC) enforced the first hours of service (HOS) rules to regulate the industry, promote the healthy development of carrier industry and protect driver's safety. Research on the safety implications of truck driver work hours were investigated in pioneering research during the 1970s (e.g., Harris and Mackie, 1972; Mackie and Miller, 1978). A major field study was undertaken in the 1990s involving drivers who drove regular routes for their firms while also taking a variety of alertness tests and being subjected to other driving measurements (e.g., Wylie et al., 1996). While the studies in the 1970s used crash and other operations data from carriers in addition to some alertness and driving indicators, the 1996 report described a study using alertness tests and measures of driving performance other than crashes. Throughout the 1990s a series of papers were published analyzing crash and non-crash data from a large, national-scale less-than-truckload (LTL) carrier (Jovanis, Kaneko and Lin, 1992; Kaneko and Jovanis, 1992; Lin, Jovanis and Yang, 1993; Lin, Jovanis and Yang, 1994). A subsequent paper (Park, Mukherjee, Gross and Jovanis, 2005) compared findings from an analysis of the 
crash data set from the 1980s and the experimental data collected by Wylie et al. Campbell conducted a study of fatigue and crash odds using fatal crash data from 1991-2002 (Campbell, 2005). Despite a substantial literature, questions remain concerning the relationship between crash occurrence and driving hours. This paper attempts to contribute to a better understanding of this relationship.

\section{METHODOLOGY}

This section describes how the basic data available from trucking companies was processed to capture the required driving descriptors, refer to Kaneko and Jovanis (1992); Lin, et al. (1993); Lin, et al. (1994); Singer and Willett (2003); and Jovanis et al., (2011) for more details. One of the primary goals of this study is to determine the effects of rest breaks on crash risk in terms of hours of driving. The response variable can be translated into time-to-failure, where crash occurrence and the time duration between the onset of operation and crash occurrence are of interest. In addition, rest breaks are time-varying covariates. Hence, survival analysis is wellsuited for this study.

Discrete-time hazard, $h_{i j}$, is defined as the conditional probability that driver $i$ will experience a crash in hours of driving $j, j=1,2, \ldots, 11$, given that the driver did not experience a crash prior to $j$. We denote driver $i$ 's value for each of the $P$ predictors in time period $\mathrm{j}$ as the vector $z_{i j}=\left[z_{1 i j}, z_{2 i j}\right.$, $\left.\ldots, z_{p i j}\right]$, and hence

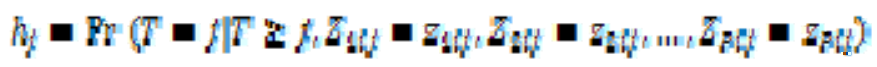

Since $h_{i j}$ are probabilities, they can be reparameterized using a logistic formula:

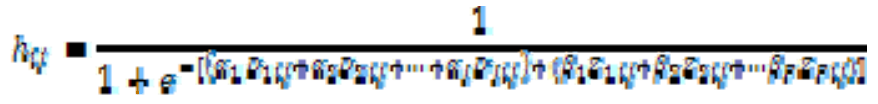

where $\left[D_{1 i j}, D_{2 i j}, \ldots, D_{J i j}\right]$ are a sequence of dummy variables, with values $\left[d_{1 i j}, d_{2 i j}, \ldots, d_{J i j}\right]$ indexing hours of driving. $J$ refers to the last hours of driving observed for anyone in the sample. The intercept parameters $\left[\alpha_{1}, \alpha_{2}, \ldots, \alpha_{J}\right]$ capture the baseline level of hazard in each hour of driving, and the slope parameters $\left[\beta_{1}, \beta_{2}, \ldots, \beta_{P}\right]$ describe the effects of the predictors, either time-varying or time-independent, on the baseline hazard function. For driver $i$ who encountered a crash in hours of driving $j_{i}$ but the crash did not occur in the first hour of driving through $j_{i}-1$, Equation (1) can be written as

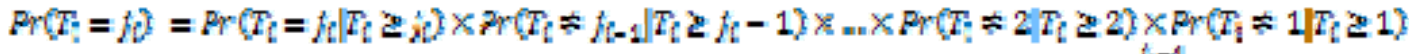

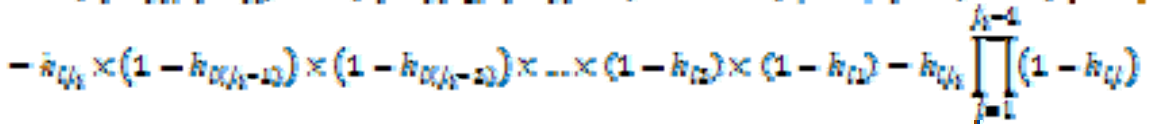

The $\left(1-h_{i j}\right)$ term in Equation (3) shows the need of data replications for periods without a crash occurrence for each individual driver $i$. Similarly, for driver who did not encounter a crash, referred to as censored in survival analysis, the probability of no crash occurrence given $j$ hours of driving is 


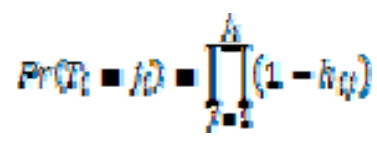

Therefore, using Equation (3) and (4), the likelihood function can be described as

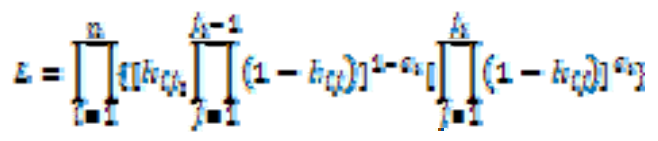

where $n$ indicates the total drivers included in this study, $\left(1-c_{i}\right)$ indicates a driver who encountered a crash during the trip, and $c_{i}$ indicates a driver who did not encounter a crash during the trip.

\section{THE DATA}

Several data sets were merged to form the full data set for this study. Data from less-thantruckload (LTL) carriers collected in 2004-05 were combined with additional data from carriers in 2010 to enhance estimation efficiency. Since the HOS rules were the same in these two time periods (except for a change in required time in sleeper berths) this step is reasonable. Moreover, a counter-part of the Chow test (Greene, 2003), was used to test the structural difference between 2010 and 2004 data and there was no evidence indicating that it was inappropriate to combine the 2004 and 2010 data (Jovanis et al., 2011). An indicator variable for year of observation will be included in analysis to see if there was a significant difference across the years. The sources of the HOS data are carrier electronic or paper files either retained by the carrier or a third party. Data are based on the driving logs that record the driver duties every 15 minutes. As in previous studies (e.g. Kaneko and Jovanis, 1992) data on domicile terminal and month of crash are used to identify two non-crash drivers. This is a case-control formulation, as shown in Table 1. All of the carriers involved in the study were large national-scale carriers. They might be characterized as being representatives of the trucking industry that are organized to generally adhere to the existing hours-of-service policies in effect at the time. While some may argue that carriers may selectively report crashes, it is difficult to see how they could selectively report crashes due to hours of service. Similar arguments could be raised about the non-crash data, but it is difficult to believe that the carriers would be able to manipulate the data to achieve a specific outcome, given the complexity of the statistical methods used. While it is possible that manipulation of the data has occurred, the authors believe it is unlikely.

Table 1. Sample Size of Data Sets Used in Study

\begin{tabular}{cccc}
\hline & Crash & Non-Crash & Total \\
\hline Carrier 1 (2004-05) & 45 & 90 & 135 \\
Carrier 2 (2004-05) & 79 & 189 & 268 \\
Carrier 3 (2010) & 105 & 188 & 293 \\
Total & 229 & 467 & 696 \\
\hline
\end{tabular}




\section{DATA ANALYSIS}

Table 2 summarizes the results of the model estimating the effects of driving time, driving breaks, and recovery period on motor carrier crash risk. A driving break is defined as drivers either being in the sleeper berth or off-duty, and the recovery period is defined as drivers having at least consecutively 34 hours of off-duty time (possibly combined with sleeper berth time) immediate prior to the trip.

\section{Effect of Driving Time and Rest Breaks}

The goodness of fit in the bottom of Table 2 shows the appropriateness of this model specification. Using the first hour as the baseline, one sees that there is an inability to detect a driving time effect for $3^{\text {rd }}$ to $5^{\text {th }}$ hour. The $2^{\text {nd }}$ hour is marginally lower than the first (using a significance probability, p, of 0.20$)$. After the $4^{\text {th }}$ hour, there is a persistent increase in crash odds with hours driving. The effect in the $6^{\text {th }}$ hour is marginally different than $1^{\text {st }}$ hour (p-value $=$ 0.096), and it shows an increase in the crash odds of being involved in a crash by 64 percent (see column 5). Hours 6 through 11 show continuous increases in the odds ratio. These results are consistent with previous studies of LTL carriers conducted with data from the 1980's (e.g. Lin, et. al., 1993; Lin, et. al., 1994). The trend in crash odds ratios is summarized graphically in Figure 1 (the solid line).

Table 2. Crash odds as function of driving time, rest breaks, and 34-hour restart

\begin{tabular}{lcccc}
\hline Variables & Coef. & Std. Err. & P $>$ z & Odds Ratio \\
\hline Second Hour (1 to 2 hours of driving) & -0.621 & 0.376 & 0.099 & 0.54 \\
Third Hour (2 to 3 hours of driving) & -0.144 & 0.331 & 0.664 & 0.87 \\
Fourth Hour (3 to 4 hours of driving) & -0.303 & 0.350 & 0.386 & 0.74 \\
Fifth Hour (4 to 5 hours of driving) & 0.248 & 0.307 & 0.419 & 1.28 \\
Sixth Hour (5 to 6 hours of driving) & 0.495 & 0.298 & 0.096 & 1.64 \\
Seventh Hour (6 to 7 hours of driving) & 0.733 & 0.292 & 0.012 & 2.08 \\
Eighth Hour (7 to 8 hours of driving) & 1.075 & 0.288 & 0.000 & 2.93 \\
Ninth Hour (8 to 9 hours of driving) & 1.346 & 0.297 & 0.000 & 3.84 \\
Tenth Hour (9 to 10 hours of driving) & 1.759 & 0.336 & 0.000 & 5.81 \\
Eleventh Hour (10 to 11 hours of driving) & 2.210 & 0.543 & 0.000 & 9.12 \\
Drivers took one break (off-duty or sleeper berth) during a trip & -0.630 & 0.173 & 0.000 & 0.53 \\
Drivers took two breaks (off-duty or sleeper berth) during a trip & -0.506 & 0.228 & 0.027 & 0.60 \\
Drivers took three or more breaks (off-duty or sleeper berth) during a trip & -0.257 & 0.137 & 0.060 & 0.77 \\
A Recovery period immediate prior to a trip starting at daytime & 0.816 & 0.273 & 0.003 & 2.26 \\
A Recovery period immediate prior to a trip starting at nighttime & 0.459 & 0.239 & 0.055 & 1.58 \\
Trip starting at nighttime without a recovery period immediate prior to the trip & 0.323 & 0.193 & 0.094 & 1.38 \\
Data collected in 2010 & -0.144 & 0.144 & 0.315 & 0.87 \\
Constant & -3.577 & 0.288 & 0.000 & NA \\
\hline
\end{tabular}

Standard error adjusted for repeated measures; Starting log-likelihood = -937.58462; Convergent log-likelihood = -879.15917; Wald chi2(17) $=147.59$, p-value $=0.000$; Pseudo $\mathrm{R} 2=0.0623$

The benefits of driving breaks depend on the number of breaks taken, as shown in Table 2 and Figure 1 (left panel). In terms of the same amount of driving time, drivers who took a single driving break experienced a 47 percent reduction in crash odds compared to drivers who did not take any break. Drivers who took two rest breaks have a 40 percent reduction in crash odds; 
those with three or more breaks a 23 percent reduction. But the difference between one and two breaks, and between two and three or more breaks are not statistically significant. This finding suggests that at least one rest break is beneficial to drivers.
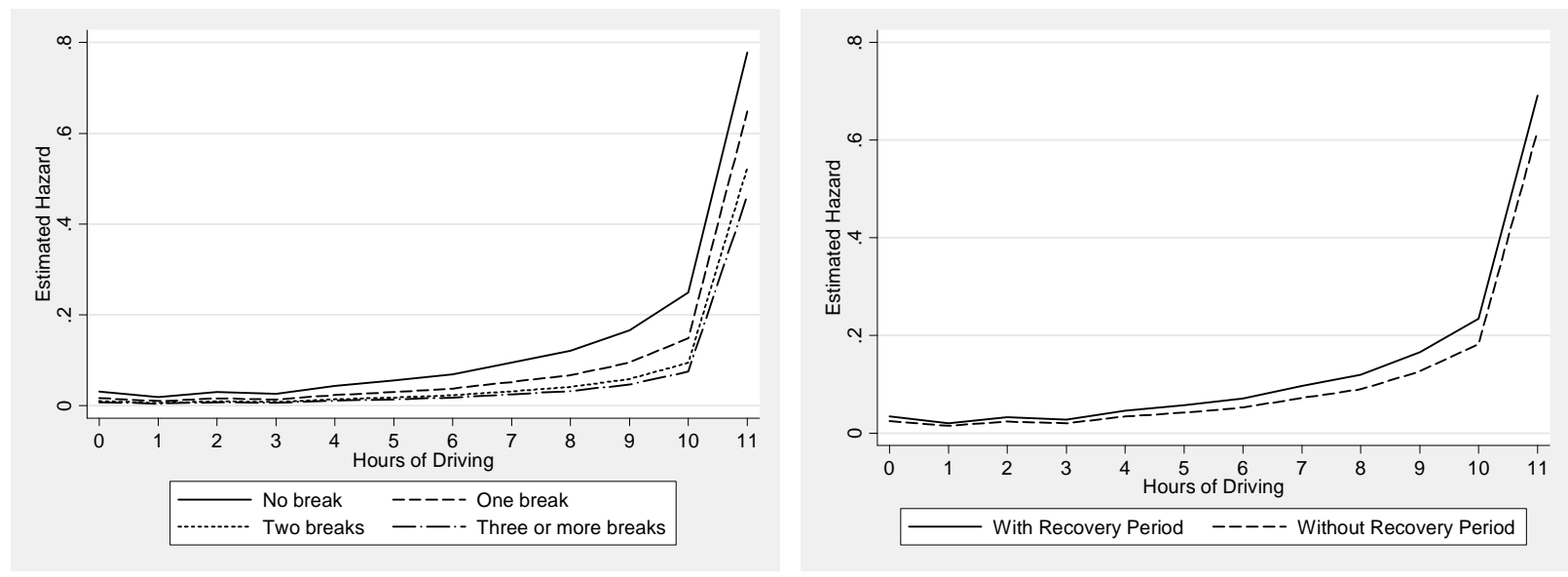

Figure 1. Hazard in terms of driving time and rest breaks (left); Hazard in terms of driving time and recovery period (right)

\section{Effect of 34-Hour Recovery Period}

Intuitively, for drivers who gain a full recovery during the recovery period, rest breaks may not be as critical as to drivers who do not. Inclusion of a recovery period variable showed that the effects of rest breaks on crash risk reduction remain unchanged. Nevertheless, an apparent counter-intuitive result has been obtained; drivers who just return from a recovery period have higher crash odds than drivers who do not, as shown in Figure 1 (right panel). Three factors could be confounding the analysis, affecting this result. Drivers may not be "resting during the recovery, so the condition to drive (unknown in this study) may be influenced by the recovery period. The pattern of driving prior to the recovery and the time when asked to return to work are two additional factors. The recovery variable was re-formulated to better identify the conditions under which the driver returned to work.

The revised model includes variables of a recovery period immediate prior to a trip starting at daytime, a recovery period immediate prior to a trip starting at nighttime, and a trip starting at nighttime without a recovery period immediate prior to the trip, compared to the baseline where a trip starts at daytime without a recovery period immediate prior. The Nighttime condition was defined as a trip that starts between 6:00 PM to 6:00 AM. It was found that all three conditions have a higher crash risk than the baseline where the trip starts during the daytime without a recovery period immediate prior to the trip, as shown in Table 2. Based on a Wald test, the effect of a recovery period immediate prior to a trip is not significantly different for the daytime and nighttime conditions ( $\mathrm{p}$-value $=0.199$ ). Moreover, the nighttime effect is not significantly different for whether there is recovery period immediate prior to a trip ( $\mathrm{p}$-value $=0.489$ ). A variety of recovery period lengths were also tested and included in the model and no evidence was found to support the argument that the recovery period lengths are related to crash risk. The critical issue is that we cannot determine exactly how operators are spending their recovery period. 
Cluster analysis was applied to identify similar driving patterns. There are 10 clusters identified in terms of drivers' on-duty, on-duty not driving, off-duty time, and time in sleeper berth, (Jovanis et al., 2011). An examination of the clusters revealed an interesting trend that supports the modeling results concerning the recovery period findings. Though there was still no statistical evidence found associated with the effect of recovery period and driving patterns, an interesting finding was noticed. As shown in Error! Reference source not found., the cluster in which drivers have similar driving pattern (regular work time centered around midnight and ending near noon) and just return from a recovery period was selected as the baseline cluster (bottom of figure). For the second cluster, where drivers also have similar driving pattern and have returned from a recover period for two days, it was found that the RR compared to the first cluster is 0.8. Similarly, the drivers in the third cluster have returned from a recovery period for three days, and the drivers in the fourth cluster have returned from a recovery period for four days. The RRs for the third and fourth cluster are 0.62 and 0.58 respectively. These graphs suggest that drivers adapt to the return to work over multiple days following a recovery period. The odds of a crash are highest on the first day back and decline as the driver continues to work. While this appears to occur for this set of night and early morning LTL drivers in this data set, additional empirical exploration is needed to validate the result.
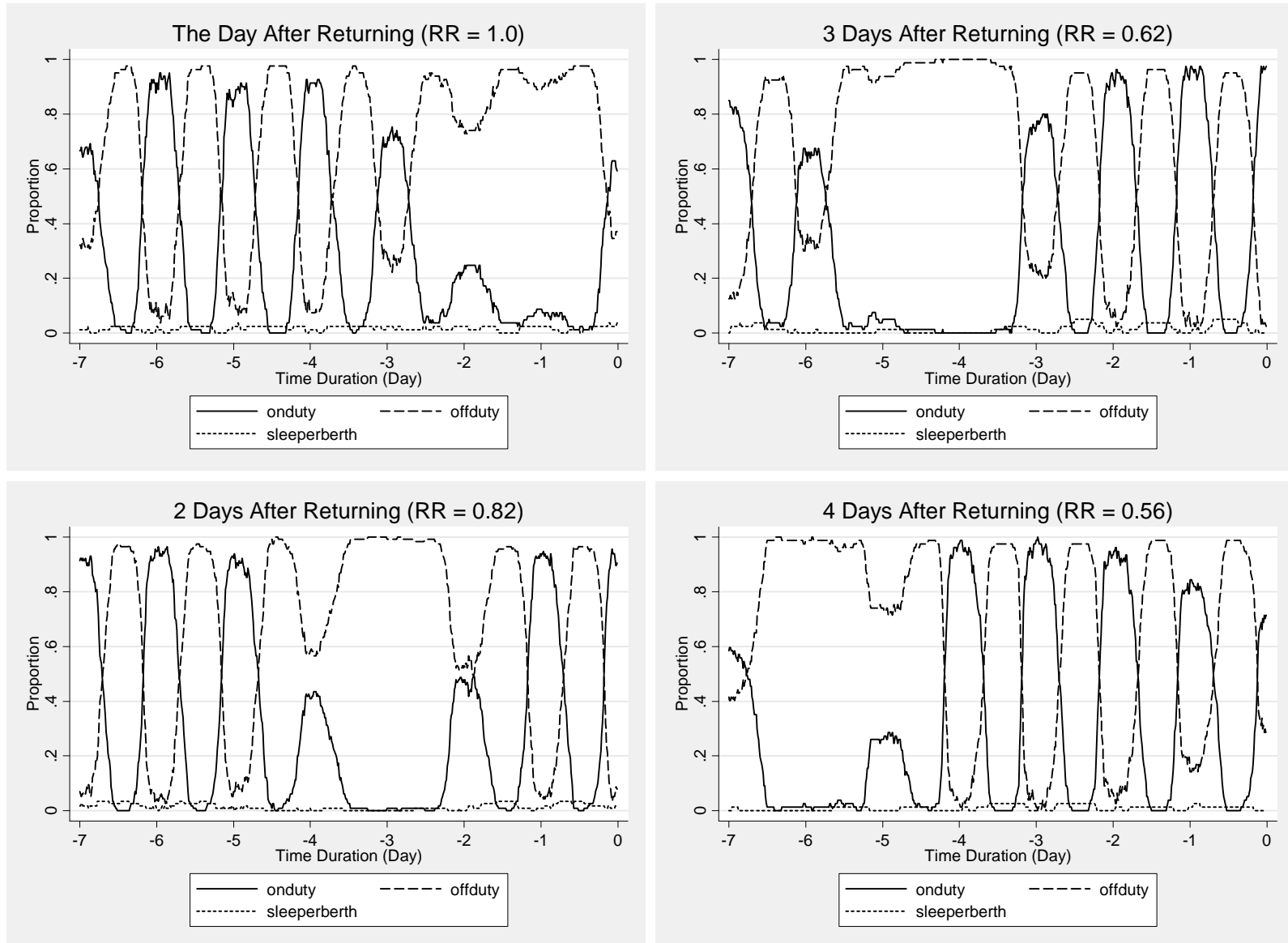

Figure 2. Cluster analysis for driving patterns 


\section{CONCLUSION AND DISCUSSION}

For this data set, the crash hazard shows a significant increase with duration of driving after the sixth hour of driving. Driving breaks reduce crash risk in terms of hours of driving. The effect of a recovery period is unclear, and needs to be further studied. For night and early morning drivers, the return from the recovery period poses the highest odds of a crash; as drivers continue to drive, the crash odds decline. It thus appears that drivers may need some transition when returning from a long off-duty period.

\section{ACKNOWLEDGEMENTS}

This paper utilized data assembled during a recently completed FMCSA project (Jovanis et al., 2011). The authors gratefully acknowledge the financial support of FMCSA in the conduct of the study. The analyses conducted in this paper were not within the scope of the FMCSA project; the findings and interpretations of the research are solely those of the authors, not FMCSA.

\section{REFERENCES}

Campbell, K., and Hwang, H.L. (2005), Estimates of the Prevalence and Risk of Fatigue in Fatal Accidents Involving Medium and Heavy Trucks from the 1991-2001 TIFA Files, Center for transportation Analysis, Oak Ridge national Laboratory, Oak Ridge, Tennessee.

Greene, W. (2003), Econometric Analysis, Fifth edition, Prentice Hall, New York.

Harris, W., R. R. Mackie, (1972), A study of the relationship among fatigue, hours of service, and safety of operations of truck and bus drivers, Final Report, BMCS RD 71-2, Washington: U.S. Department of Transportation, Federal Highway Administration, Bureau of Motor Carrier Safety.

Jovanis, P.P., Kaneko, T., and Lin, T. (1991), Exploratory Analysis of Motor Carrier Accident Risk and Daily Driving Pattern, Transportation Research Record 1322, Transportation Research Board, Washington, D.C., pp. 34-43.

Jovanis, P.P., K.F. Wu, C. Chen, (2011). Hours of Service and Driver Fatigue: Driver Characteristic Research. Washington, DC: Federal Motor Carrier Safety Administration, FMCSA-RRR-11-018.

Kaneko, T., P.P. Jovanis, (1992), Multiday driving Patterns and Motor Carrier Accident Risk: A Disaggregate Analysis. Accident Analysis and Prevention, Vol. 24, No. 5, pp. 437-456.

Lin, T.D., P.P. Jovanis, C.Z. Yang, (1993) Modeling the Effect of Driver Service Hours on Motor Carrier Accident Risk Using Time Dependent Logistic Regression, Transportation Research Record 1407, Transportation Research Board, Washington, D.C., pp. 1-10.

Lin, T.D., P.P. Jovanis, C.Z. Yang, (1994), Time of Day Models of Motor Carrier Accident Risk, Transportation Research Record 1467, Transportation Research Board, Washington, D.C., pp. 1-8.

Mackie, R.R., and Miller, J.C. (1978), Effects of hours of service regularity of schedules, and cargo loading on truck and bus driver fatigue, DOT Report HS-5-01142. 
Park, S-W, Mukherjee, A., Gross, F., and Jovanis, P.P. (2005), "Safety Implications of Multi-day Driving Schedules for Truck Drivers: Comparison of Field Experiments and Crash Data Analysis”, Journal of the Transportation Research Board, No. 1922, pp. 167-174.

Singer, J.D., J.B. Willett, (2003). Applied Longitudinal Data Analysis. New York: Oxford.

Wylie, D.D., Shultz, T., Miller, J.C., Mitler, M.M., and Mackie, R.R. (1996), Commercial Motor Vehicle Driver fatigue and Alertness Study: Technical Summary, Publication FHWA-MC-97001. FHWA, U.S. Department of Transportation. 